\title{
Rodent Lethality Models Are Problematic for Evaluating Antivenoms for Human Envenoming
}

\author{
Anjana Silva ${ }^{1,2,3}$, Wayne C. Hodgson ${ }^{2}$, Theo Tasoulis ${ }^{4}$ and Geoffrey K. Isbister ${ }^{3,4 *}$ \\ ${ }^{1}$ Department of Parasitology, Faculty of Medicine and Allied Sciences, Rajarata University of Sri Lanka, Saliyapura, Sri Lanka, \\ ${ }^{2}$ Monash Venom Group, Faculty of Medicine, Nursing and Health Sciences, Monash University, Clayton, VIC, Australia, ${ }^{3}$ South \\ Asian Clinical Toxicology Research Collaboration, University of Peradeniya, Peradeniya, Sri Lanka, ${ }^{4}$ Clinical Toxicology Research \\ Group, University of Newcastle, Newcastle, NSW, Australia
}

Keywords: lethality, snake venom, antivenom, LD50, ED50, rodent, mouse

\section{INTRODUCTION}

Snakebite is a major public health problem in the tropics, being closely associated with agricultural economy, poverty and underdevelopment. The neglected nature of the condition over a long period of time has meant that our current understanding of the epidemiology, clinical effects, pathophysiology and the treatment of snakebite has many gaps (Isbister and Silva, 2018). Knowledge of the pathophysiology is essential to understanding the clinical effects of snake envenoming and, more importantly, the response to antivenom.

Snake venoms are a complex mix of different toxins with varying pharmacological properties

OPEN ACCESS

Edited by:

Jing-Lin Wang,

Beijing Institute of Microbiology and Epidemiology, China

Reviewed by:

Dexter Tagwireyi,

University of Zimbabwe, Zimbabwe

*Correspondence:

Geoffrey K. Isbister geoff.isbister@gmail.com

Specialty section:

This article was submitted to Pharmacology of Ion Channels and Channelopathies, a section of the journal Frontiers in Pharmacology

Received: 07 December 2021 Accepted: 17 January 2022

Published: 03 February 2022

Citation:

Silva A, Hodgson WC, Tasoulis T and Isbister GK (2022) Rodent Lethality Models Are Problematic for Evaluating Antivenoms for Human Envenoming.

Front. Pharmacol. 13:830384. doi: 10.3389/fphar.2022.830384
(Tasoulis and Isbister, 2017). The effects of snake venoms on natural prey species are likely to be different to the effects on non-prey species, such as humans (Richards et al., 2012). Animal models have been important tools for understanding the effects of snake venoms on humans. The action of the whole venom and isolated toxins, and their response to antivenoms, are tested in living laboratory animals, such as rats, mice, and rabbits (in-vivo) and tissues isolated from laboratory animals, such as rats, mice, rabbits, frogs, and chickens (in-vitro) (Silva and Isbister, 2020). Despite the biochemical and functional complexity of snake venoms observed in animal models, bites by medically important snakes commonly result in a narrow range of acute effects in human envenoming, including local tissue necrosis, venom-induced consumption coagulopathy (VICC), neuromuscular paralysis, acute kidney injury (AKI) and myotoxicity. It is important that animal models used for testing snake toxins, venoms and antivenoms, to better represent human envenoming, mirror the observed clinical effects of human envenoming.

Neutralisation of rodent lethality from snake venoms in-vivo has been recommended to manufacturers and regulatory bodies as an essential pre-clinical test of antivenoms by the World Health Organisation (WHO). Mouse lethality prevention assays are considered the "gold standard test" until alternative tests become accepted (World Health Organisation, 2010; Calvete et al., 2018). The WHO further states that the suffering of test animals due to the venom effects until death, or during the 24 or 48 observation period, in the lethality studies outweighed by the larger benefits to humans (World Health Organisation, 2010).

\section{Why Rodent Lethality Models Are Problematic for Evaluating Antivenoms for Human Envenoming?}

The majority of snake venoms that cause paralysis in envenomed humans contain pre-synaptic neurotoxins, which cause paralysis that is, not reversible with antivenom (Silva et al., 2017, 2018). The other major group of snake neurotoxins are the post-synaptically acting $\alpha$-neurotoxins. These toxins are present in venoms from a range of snakes, including many species that do not result in 
paralysis in humans (Barber et al., 2013; Youngman et al., 2021). The proportion of $\alpha$-neurotoxins in snake venoms is known to be associated with the prey selectivity of the venoms (Harris et al., 2020), which further suggests that these particular toxins are animal-specific. The two major types of $\alpha$-neurotoxins, i.e., longchain (LaNTx) and short-chain (SaNTx), bind to the same region of the $\alpha$-subunit of the $\mathrm{nAChR}$, resulting in inhibition of neurotransmission at the skeletal neuromuscular junction. Current knowledge about the relevance of snake venom $a$ neurotoxins in human envenoming and paralysis is largely based on projections from data generated from rodent, avian, and amphibian pharmacological models (Silva et al., 2017). However, using skeletal muscle from several animals, including rodents, non-human primates and humans, a largely neglected study, dating back to 1985 , showed that human nAChR have an exceptionally low affinity for SaNTx compared to LaNTx, while both groups bind with high affinity to mouse nAChRs (Ishikawa et al., 1985). Consistent with these observations, in a functional study using human and rat nAChR, we recently demonstrated that the human nAChR is more resistant to snake SaNTx compared to the rat nAChR, as evidenced by marked differences in the speed of the reversibility of toxin-mediated inhibition of the human nAChR (Silva et al., 2018). The experiments, with and without various mutations of the human and rat $\mathrm{nAChR}$, showed that this species difference is due to the absence of large aromatic amino acid residues at positions 187 and 189 in loop $\mathrm{C}$ of the a subunit of the human nAChR. This is in contrast to rats, mice, and nAChRs in other animals commonly used for in-vitro testing, including Torpedo californica, which possess large aromatic amino acid residues at equivalent positions. However, no such difference was observed between the effects of LaNTx on rat and human nAChRs.

Inhibition of the $\mathrm{nAChR}$ only requires the binding of one $\alpha$ neurotoxin molecule to one of the two ACh binding sites on the receptor. Theoretically, paralysis could develop in humans following a snakebite if the concentration of $\alpha$-neurotoxins at the neuromuscular junction is such that the nAChRs are inhibited and $\alpha$-neurotoxin-receptor binding could be sustained, without being rapidly reversed naturally. However, sustaining sufficient $\alpha$ neurotoxin concentrations at the neuromuscular junction to maintain inhibition of the $\mathrm{nAChR}$ depends on the relative abundance of the $\alpha$-neurotoxins in the particular snake venom as well as the amount of venom injected during the snakebite. This means that snakes injecting large amounts of venom when biting humans, and/or having a higher relative abundance of LaNTx in their venoms, such as some species of cobras (Naja) or the king cobra (Ophiophagus hannah), can potentially cause postsynaptic neurotoxic paralysis in humans. Snakes containing only SaNTx or only small amounts of LaNTx, will not cause paralysis in humans. SaNTxs are unlikely to be clinically important, except in unique situations such as Philippine cobra (Naja philippinensis) envenomings in which there is such a large venom load, human paralysis can still occur, despite a higher relative abundance of SaNTx ( 45\%) (Tan et al., 2019). Therefore, assays heavily influenced by the effects of $a$ neurotoxins in any animal except humans are not representative of paralysis in humans and are problematic for antivenom development. In agreement with this, recent studies have demonstrated that the outcome of rodent lethality assays are heavily influenced by the $\alpha$-neurotoxin activity of snake venoms, when $\alpha$-neurotoxins are present in the venoms (Petras et al., 2011; Pruksaphon et al., 2020).

Rodent lethality prevention assays have shown results comparable to human envenoming in some viperine venoms lacking a-neurotoxins, such as carpet vipers (Echis sp.) and south American Bushmasters (Lachecis sp.) (Gutiérrez et al., 1988; Bard et al., 1994; Diniz-Sousa et al., 2020). Although $\alpha$-neurotoxins are mostly found in elapids and colubrids, they are present even in some viperid venoms that do not cause neurotoxicity in humans, such as Puff adders (Wang et al., 2020; Youngman et al., 2021) hence the utility of these assays remains narrow.

There are other examples in which animal models do not represent human envenoming. Plasma from several large animals, including rats, were shown to be resistant to concentrations of procoagulant toxins several orders of magnitude greater compared to humans, from viperids such as Daboia russelii, Echis carinatus, Callocellasma rhodostoma and elapids such as Pseudonaja textillis, (Maduwage KP. et al., 2016). This means, in order to replicate VICC in rodent models, the animals need to be exposed to very high venom concentrations, which would lead to the death of animals from the primary and secondary effects of venom that are not relevant to humans (i.e., neurotoxicity). In a recent study, acute kidney injury was not able to be replicated in rats with sub-lethal doses of D. russelii venom suggesting it to be a poor model for venom-induced acute kidney injury (Wijewickrama et al., 2018). VICC, AKI and thrombocytopaenia only occurred with experimental venom doses that were unrealistically higher than what is observed in actual human envenomings, making it difficult to match the reallife envenoming scenarios in humans (Tan et al., 2012; Romanelli et al., 2021; Thomazini et al., 2021; Yamamoto et al., 2021).

\section{DISCUSSION}

Among the WHO approved list of essential quality control parameters for routine quality control testing of antivenoms, the sole parameter that is used to assess the pharmacological/ therapeutic efficacy of antivenom is the lethality neutralisation test (World Health Organisation, 2017; Patra et al., 2021). The WHO guidelines for the production, control and regulation of snake antivenom immunoglobulins, published in 2017, states that "Despite reservations about the physiological relevance of these animal (murine) models to human envenoming and the harm that these in vivo assays cause to the animals, they are used by both manufacturers and regulatory authorities worldwide for determining venom lethality $\left(\mathrm{LD}_{50}\right)$ and antivenom neutralizing capacity $\left(\mathrm{ED}_{50}\right)$ as these are currently the only validated means of assessing venom toxicity and antivenom neutralizing potency" (World Health Organisation, 2017). Further, it states "Non-sentient or in vitro assays as alternatives to the standard venom $\mathrm{LD}_{50}$ and antivenom $\mathrm{ED}_{50}$ in vivo tests have been promoted. Unfortunately, such systems have not been developed to the point where they can fully replace 
the above-mentioned preclinical assays." Further, the WHO report states that "in the absence of effective alternatives, the continued use of experimental animals is still justified by the considerable benefits to human health of these preclinical assays" (World Health Organisation, 2017).

The fundamental assumption behind testing venoms, toxins and antivenoms, using rodent lethality models in relation to human envenoming, is that the "toxins possessing the highest rodent lethality are the most medically important toxins in human envenoming" (Lauridsen et al., 2016; Calvete et al., 2018; Silva and Isbister, 2020). This assumption requires evidence that there is a clear relationship between the outcome of rodent lethality tests and clinical toxicity in human envenoming, in venom doses comparable with bites in human envenomings. However, due to the reasons highlighted above, we argue that the relevance of the death or survival of an animal observed in rodent lethality studies, lethality prevention studies or rescue models, to an envenomed human with snakebite is highly questionable. It is not an appropriate model for characterisation of medically important venoms and preclinical testing of snake antivenoms. Rodent lethality-based assays cause enormous suffering to the test animals, so the minimal value of these lethality models does not outweigh the suffering of test animals.

The venom composition of snakes varies enormously resulting in different envenoming syndromes in envenomed humans with different clinical outcomes. Reducing the complexity of the effects of snake venoms in humans to a single parameter, the lethality of an experimental animal, is a gross oversimplification. Efficacy assays need to be based on the ability of the antibodies in antivenoms to bind with the venom toxins in-vitro and to neutralise or prevent the clinically relevant effects of snake toxins in human envenoming (Maduwage K. et al., 2016). The efficacy of antivenoms in neutralising the clinically mostimportant systemic effects of envenoming such as neuromuscular paralysis, VICC and myotoxicity could be successfully tested using in-vitro functional assays such as

\section{REFERENCES}

Barber, C. M., Isbister, G. K., and Hodgson, W. C. (2013). Alpha Neurotoxins. Toxicon 66, 47-58. doi:10.1016/j.toxicon.2013.01.019

Bard, R., Lima, J. C. R. d., Sa Neto, R. P. d., Oliveira, S. G. d., and Santos, M. C. d. (1994). Ineficácia Do antiveneno botrópico na neutralização da atividade coagulante Do veneno de Lachesis muta muta: relato de caso e comprovação experimental. Rev. Inst. Med. Trop. S. Paulo 36, 77-81. doi:10. 1590/s0036-46651994000100012

Calvete, J. J., Rodríguez, Y., Quesada-Bernat, S., and Pla, D. (2018). Toxin-resolved Antivenomics-Guided Assessment of the Immunorecognition Landscape of Antivenoms. Toxicon 148, 107-122. doi:10.1016/j.toxicon.2018.04.015

Diniz-Sousa, R., Moraes, J. D. N., Rodrigues-da-Silva, T. M., Oliveira, C. S., and Caldeira, C. A. D. S. (2020). A Brief Review on the Natural History, Venomics and the Medical Importance of Bushmaster (Lachesis) Pit viper Snakes. Toxicon $X$ 7, 100053. doi:10.1016/j.toxcx.2020.100053

Gutiérrez, J. M., Chaves, F., Rojas, E., Elizondo, J., Avila, C., and Cerdas, L. (1988). Production of Monovalent Anti-Bothrops asper Antivenom: Development of Immune Response in Horses and Neutralizing Ability. Rev. Biol. Trop. 36, 511-517. neuromuscular preparations that test the neutralisation of clinically relevant pre-synaptic neurotoxins and post-synaptic toxins by antivenom (Silva et al., 2016b; 2016a) and in-vitro procoagulant activity neutralisation assays that test the ability of antivenoms to neutralise the procoagulant effects of venoms on human plasma (Maduwage K. et al., 2016). Although the in-vitro procoagulant assays are relatively simple to conduct, the in-vitro neurotoxicity assays require organ-bath systems and sufficient technical expertise. More ethically appropriate alternative tests such as embryonated egg models have also been introduced, but need validation across a range of snake venoms as well as the relevance to envenoming syndromes in humans (Sells, 2003; Verity et al., 2021).

Rodent lethality models are relatively simple to conduct hence not resource-intensive. They are easy to interpret and are affordable for developing settings (1). While acknowledging the long history and simplicity of rodent $\mathrm{LD}_{50}$ and $\mathrm{ED}_{50}$ assays, we emphasise that they measure toxic effects that are not necessarily relevant in humans. They do not sufficiently represent envenoming syndromes in humans and have considerable ethical issues. The lack of alternative models does not justify the use of irrelevant and ethically questionable rodent lethality tests.

\section{AUTHOR CONTRIBUTIONS}

Conceived the work: AS, GI, and WH; Drafted the article: AS, Critically revised the manuscript: GI, WH, and TT; Acquired funding: GI.

\section{FUNDING}

GI received National Health and Medical Research Council (NHMRC) Centers for Research Excellence Grant ID: 1110343 and NHMRC Senior Research Fellowship Grant ID: 1154503.

Harris, R. J., Zdenek, C. N., Harrich, D., Frank, N., and Fry, B. G. (2020). An Appetite for Destruction: Detecting Prey-Selective Binding of $\alpha$-Neurotoxins in the Venom of Afro-Asian Elapids. Toxins (Basel) 12, 1-12. doi:10.3390/ toxins 12030205

Isbister, G. K., and Silva, A. (2018). Addressing the Global challenge of Snake Envenoming. Lancet 392, 619-620. doi:10.1016/S0140-6736(18)31328-X

Ishikawa, Y., Kano, M., Tamiya, N., and Shimada, Y. (1985). Acetylcholine Receptors of Human Skeletal Muscle: a Species Difference Detected by Snake Neurotoxins. Brain Res. 346, 82-88. doi:10.1016/0006-8993(85)91097-2

Lauridsen, L. P., Laustsen, A. H., Lomonte, B., and Gutiérrez, J. M. (2016). Toxicovenomics and Antivenom Profiling of the Eastern green Mamba Snake (Dendroaspis Angusticeps). J. Proteomics 136, 248-261. doi:10.1016/j. jprot.2016.02.003

Maduwage, K., Silva, A., O’Leary, M. A., Hodgson, W. C., and Isbister, G. K. (2016b). Efficacy of Indian Polyvalent Snake Antivenoms against Sri Lankan Snake Venoms: Lethality Studies or Clinically Focussed In Vitro Studies. Sci. Rep. 6, 26778. doi:10.1038/srep26778

Maduwage, K. P., Scorgie, F. E., Lincz, L. F., O’Leary, M. A., and Isbister, G. K. (2016a). Procoagulant Snake Venoms Have Differential Effects in Animal Plasmas: Implications for Antivenom Testing in Animal Models. Thromb. Res. 137, 174-177. doi:10.1016/j.thromres.2015.12.002 
Patra, A., Herrera, M., Gutiérrez, J. M., and Mukherjee, A. K. (2021). The Application of Laboratory-Based Analytical Tools and Techniques for the Quality Assessment and Improvement of Commercial Antivenoms Used in the Treatment of Snakebite Envenomation. Drug Test. Anal. 13, 1471-1489. doi:10.1002/dta.3108

Petras, D., Sanz, L., Segura, A., Herrera, M., Villalta, M., Solano, D., et al. (2011). Snake Venomics of African Spitting Cobras: Toxin Composition and Assessment of Congeneric Cross-Reactivity of the Pan-African EchiTAbPlus-ICP Antivenom by Antivenomics and Neutralization Approaches. J. Proteome Res. 10, 1266-1280. doi:10.1021/pr101040f

Pruksaphon, K., Tan, K. Y., Tan, C. H., Simsiriwong, P., Gutiérrez, J. M., and Ratanabanangkoon, K. (2020). An In Vitro a-neurotoxin-nAChR Binding Assay Correlates with Lethality and In Vivo Neutralization of a Large Number of Elapid Neurotoxic Snake Venoms from Four Continents. Plos Negl. Trop. Dis. 14, e0008581-21. doi:10.1371/journal.pntd.0008581

Richards, D. P., Barlow, a., and Wüster, W. (2012). Venom Lethality and Diet: Differential Responses of Natural Prey and Model Organisms to the Venom of the Saw-Scaled Vipers (Echis). Toxicon 59, 110-116. doi:10.1016/j.toxicon. 2011.10.015

Romanelli, M. A., Soeiro, P. A., Silva, R. C. D., Taveira-da-Silva, R., Melo, P. A., and Lara, L. S. (2021). Revisiting the Acute Kidney Injury in Wistar Rats Experimentally Envenomated Wity Bothrops Jararacussu Venom. Toxicon 199, 117-126. doi:10.1016/j.toxicon.2021.06.004

Sells, P. G. (2003). Animal Experimentation in Snake Venom Research and In Vitro Alternatives. Toxicon 42, 115-133. doi:10.1016/S0041-0101(03)00125-9

Silva, A., Cristofori-Armstrong, B., Rash, L. D., Hodgson, W. C., and Isbister, G. K. (2018). Defining the Role of post-synaptic a-neurotoxins in Paralysis Due to Snake Envenoming in Humans. Cell. Mol. Life Sci. 75, 4465-4478. doi:10.1007/ s00018-018-2893-x

Silva, A., Hodgson, W. C., and Isbister, G. K. (2017). Antivenom for Neuromuscular Paralysis Resulting from Snake Envenoming. Toxins (Basel) 9, E143. doi:10.3390/toxins9040143

Silva, A., Hodgson, W. C., and Isbister, G. K. (2016a). Cross-Neutralisation of In Vitro Neurotoxicity of Asian and Australian Snake Neurotoxins and Venoms by Different Antivenoms. Toxins (Basel) 8, 302. doi:10.3390/toxins8100302

Silva, A., and Isbister, G. K. (2020). Current Research into Snake Antivenoms, Their Mechanisms of Action and Applications. Biochem. Soc. Trans. 48, 537-546. doi:10.1042/BST20190739

Silva, A., Johnston, C., Kuruppu, S., Kneisz, D., Maduwage, K., Kleifeld, O., et al. (2016b). Clinical and Pharmacological Investigation of Myotoxicity in Sri Lankan Russell's Viper (Daboia russelii) Envenoming. Plos Negl. Trop. Dis. 10, e0005172. doi:10.1371/journal.pntd.0005172

Tan, C. H., Tan, N. H., Sim, S. M., Fung, S. Y., Jayalakshmi, P., and Gnanathasan, C. A. (2012). Nephrotoxicity of Hump-Nosed Pit viper (Hypnale Hypnale) Venom in Mice Is Preventable by the Paraspecific Hemato Polyvalent Antivenom (HPA). Toxicon 60, 1259-1262. doi:10.1016/j.toxicon.2012.08.012

Tan, C. H., Wong, K. Y., Chong, H. P., Tan, N. H., and Tan, K. Y. (2019). Proteomic Insights into Short Neurotoxin-Driven, Highly Neurotoxic Venom of Philippine Cobra (Naja Philippinensis) and Toxicity Correlation of Cobra Envenomation in Asia. J. Proteomics 206, 103418. doi:10.1016/j.jprot.2019. 103418
Tasoulis, T., and Isbister, G. (2017). A Review and Database of Snake Venom Proteomes. Toxins (Basel) 9, 290. doi:10.3390/toxins 9090290

Thomazini, C. M., Sachetto, A. T. A., de Albuquerque, C. Z., de Moura Mattaraia, V. G., de Oliveira, A. K., Serrano, S. M. T., et al. (2021). Involvement of von Willebrand factor and botrocetin in the thrombocytopenia induced by Bothrops jararaca snake venom. Plos Negl. Trop. Dis. 15, e0009715. doi:10.1371/journal. pntd.0009715

Verity, E. E., Stewart, K., Vandenberg, K., Ong, C., and Rockman, S. (2021). Potency Testing of Venoms and Antivenoms in Embryonated Eggs: An Ethical Alternative to Animal Testing. Toxins 13, 233-313. doi:10.3390/ toxins 13040233

Wang, C. R., Bubner, E. R., Jovcevski, B., Mittal, P., and Pukala, T. L. (2020). Interrogating the Higher Order Structures of Snake Venom Proteins Using an Integrated Mass Spectrometric Approach. J Proteomics 216, 103680. doi:10. 1016/j.jprot.2020.103680

Wijewickrama, E. S., Kurukulasooriya, I., Gunatilake, M., Priyani, A. A., Gnanathasan, A., Gawarammana, I., et al. (2018). Determination of the Sub-lethal Nephrotoxic Dose of Russell's viper (Daboia russelii) Venom in Wistar Rats. Toxicon 152, 43-45. doi:10.1016/j.toxicon.2018.07.023

World Health Organisation (2017). Guidelines for the Production, Control and Regulation of Snake Antivenom immunoglobulinsNo. 964). in Replacement of Annex 2 of WHO Technical Report Series. Geneva, Switzerland.

World Health Organisation (2010). WHO Guidelines for the Production Control and Regulation of Snake Antivenom Immunoglobulins. Geneva, Switzerland.

Yamamoto, A., Ito, T., and Hifumi, T. (2021). Attempt to Develop Rat Disseminated Intravascular Coagulation Model Using Yamakagashi (Rhabdophis Tigrinus) Venom Injection. Toxins (Basel) 13, 160. doi:10. 3390/toxins13020160

Youngman, N. J., Harris, R. J., Huynh, T. M., Coster, K., Sundman, E., Braun, R., et al. (2021). Widespread and Differential Neurotoxicity in Venoms from the Bitis Genus of Viperid Snakes. Neurotox. Res. 39, 697-704. doi:10.1007/s12640021-00330-4

Conflict of Interest: The authors declare that the research was conducted in the absence of any commercial or financial relationships that could be construed as a potential conflict of interest.

Publisher's Note: All claims expressed in this article are solely those of the authors and do not necessarily represent those of their affiliated organizations, or those of the publisher, the editors and the reviewers. Any product that may be evaluated in this article, or claim that may be made by its manufacturer, is not guaranteed or endorsed by the publisher.

Copyright (C) 2022 Silva, Hodgson, Tasoulis and Isbister. This is an open-access article distributed under the terms of the Creative Commons Attribution License (CC BY). The use, distribution or reproduction in other forums is permitted, provided the original author(s) and the copyright owner(s) are credited and that the original publication in this journal is cited, in accordance with accepted academic practice. No use, distribution or reproduction is permitted which does not comply with these terms. 